\title{
Index to the Dba' bzhed Manuscript
}

A note on the sigla used in this index: Tibetan characters provided within round brackets represent further information on the identity of

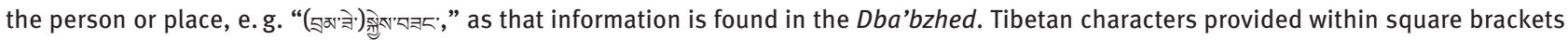

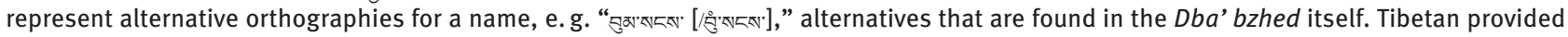

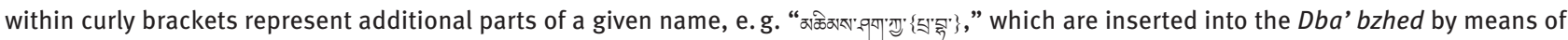
annotations.

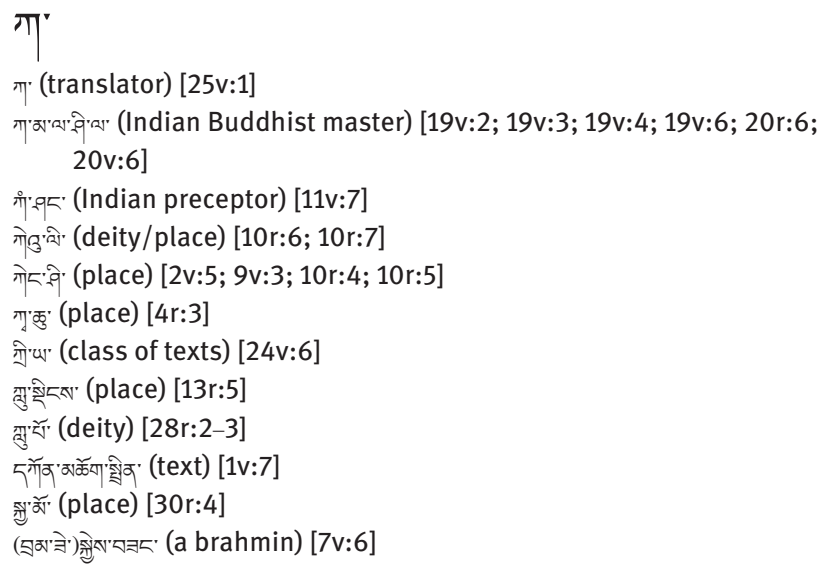

$\square^{\top}$

ब'ळे' (Kashmir) [7v:4; 7v:5; 7v:6; 8r:5]

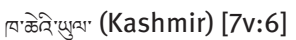

\खसब (place) [interlinear annotation above 4r:2]

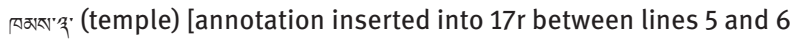
referring to $17 \mathrm{r}: 5]$

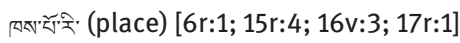

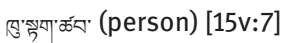

लㅐㄷ'

ब्रेंकुछ (translator) [annotation at the bottom of $17 \mathrm{v}$ referring to $17 \mathrm{v}: 2]$

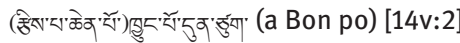

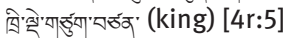

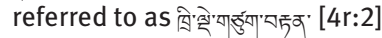
referred to only as వह์ से [4r:5]

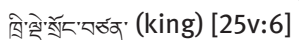
referred to only as 쥒

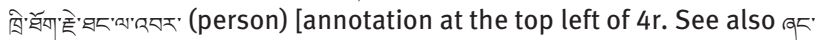

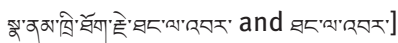

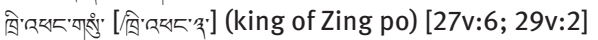

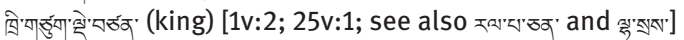

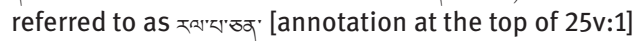
referred to as 쥑 제저 [25v:1]

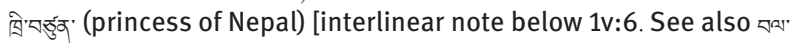

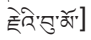

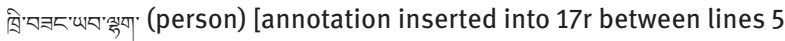
and 6 referring to $17 \mathrm{r}: 5]$

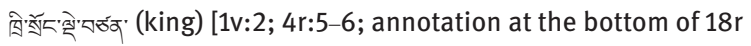
referring to $17 \mathrm{v}: 2 ; 25 \mathrm{r}: 2 ; 26 \mathrm{r}: 3 ; 26 \mathrm{r}: 7 ; 30 \mathrm{r}: 7]$

referred to as 闪:

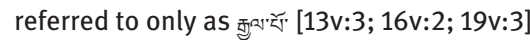

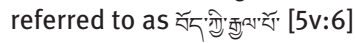

referred to only as पर्ठक से [6r:1; 6r:4; 6r:5; 6r:7; 6v:1;

$6 \mathrm{v}: 6 ; 6 \mathrm{v}: 7 ; 7 \mathrm{r}: 6 ; 7 \mathrm{r}: 7 ; 7 \mathrm{v}: 1 ; 8 \mathrm{r}: 2 ; 8 \mathrm{r}: 3 ; 8 \mathrm{v}: 2 ; 8 \mathrm{v}: 6 ; 8 \mathrm{v}: 7$;
$9 \mathrm{v}: 3 ; 9 \mathrm{v}: 4 ; 9 \mathrm{v}: 5 ; 10 \mathrm{r}: 1 ; 10 \mathrm{v}: 6 ; 11 \mathrm{v}: 1 ; 11 \mathrm{v}: 3 ; 11 \mathrm{v}: 5 ; 12 \mathrm{v}: 3$; $12 \mathrm{v}: 4 ; 13 \mathrm{r}: 6 ; 13 \mathrm{r}: 7 ; 13 \mathrm{v}: 3 ; 13 \mathrm{v}: 4 ; 13 \mathrm{v}: 5 ; 14 \mathrm{r}: 2 ; 14 \mathrm{r}: 6 ; 15 \mathrm{r}: 4$; 15r:7; 15v:2; 15v:3; 15v:4; 15v:5; 16r:4; 16r:6; 16v:1; 17r:4; annotation at the bottom right of $17 \mathrm{r}$ referring to $17 \mathrm{r}: 5$; interlinear annotation above 17v:6; 18r:3; 18v:4; 18v:6; interlinear annotation above 19r:3; interlinear annotation above 19r:6; 19v:6; interlinear annotation above 20r:1; 25r:5; 25v:5-6]

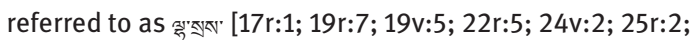
$25 r: 4 ; 26 r: 3 ; 26 r: 7 ; 30 v: 3 ; 31 r: 2 ; 31 r: 4]$

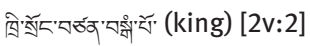

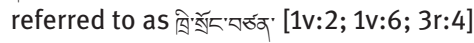

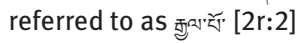

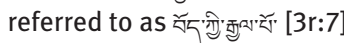

referred to as मर्ठक्टे [2r:3; 2r:4; 2r:5; 2v:2; 3r:2; 3r:3; 3v:1; $3 v: 3 ; 3 v: 4 ; 3 v: 5 ; 3 v: 6]$

referred to as 殸 वर्ठक्व [6v:2]

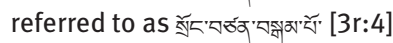

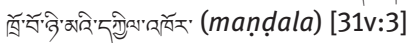

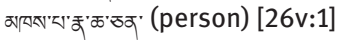

रति:

रायर' 4r:7]

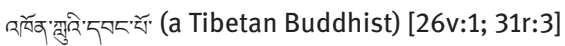

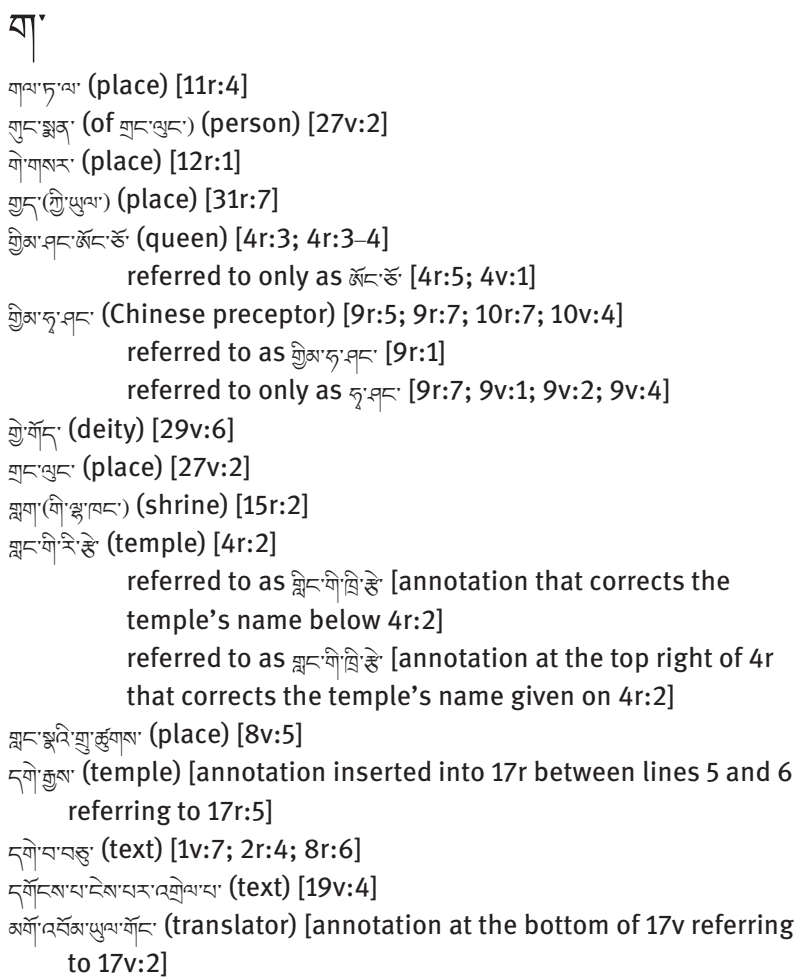




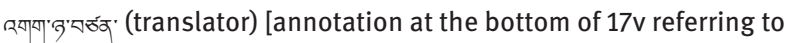
$17 \mathrm{v}: 2]$

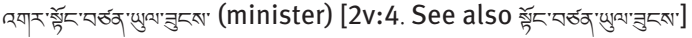

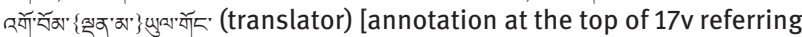
to $17 \mathrm{v}: 2$ ]

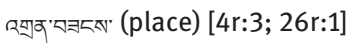

羊 5 '्रेट (castle) [29v:7]

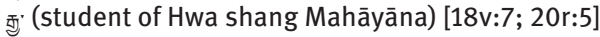

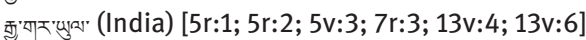

referred to as क्षुप्य [interlinear note at the top of $1 \mathrm{v}: 3 ; 1 \mathrm{v}: 5$; 1v:6; 1v:7; 2v:3; 5v:5; 7r:1; 8r:6; 11v:7; 12r:1; 12r:2; 15v:5; annotation at the top of $17 \mathrm{v}: 2 ; 22 \mathrm{v}: 4 ; 25 \mathrm{r}: 6 ; 25 \mathrm{r}: 7 ; 25 \mathrm{v}: 1$; $25 \mathrm{v}: 5 ; 28 \mathrm{r}: 1 ; 28 \mathrm{v}: 7]$

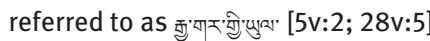

可言' (emperor of China during the reign of Khri Lde gtsug btsan)

[2v:3; 2v:5; 2v:6; 2v:7; 3r:1; 3r:2]

⿹ $9 \mathrm{v}: 3 ; 10 \mathrm{r}: 5 ; 10 \mathrm{r}: 6 ; 10 \mathrm{v}: 3]$

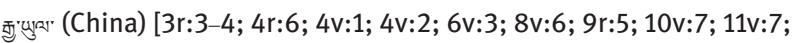
$12 \mathrm{r}: 2 ; 18 \mathrm{v}: 6 ; 25 \mathrm{r}: 7$

referred to as 䍖 [2r:2; 4r:3; 4v:1; 4v:2; annotation at the bottom of $17 \mathrm{v}$ referring to $17 \mathrm{r}: 2]$

क्रुरे' (deity/statue) [4r:6; 5r:1; 6v:3; 6v:5; 6v:6; 7r:1]

referred to as 쥫 left of $4 r$ referring to $4 r: 6 ; 5 r: 1 ; 6 v: 3 ; 8 v: 4 ; 15 r: 1]$

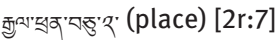

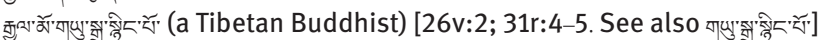

新"' (deity) [16r:5; 16r:6]

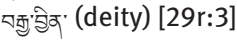

$\Sigma^{\prime}$

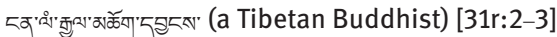

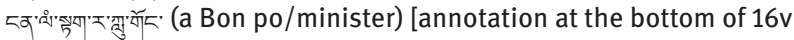
referring to $16 \mathrm{v}: 7 ; 26 \mathrm{r}: 4]$

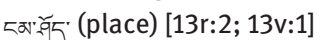

\section{$\bar{\mho}^{\prime}$}

ठัँा" (translator) [25v:1]

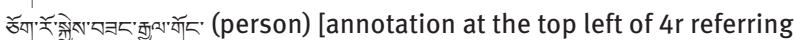
to $4 \mathrm{r}: 6 ; 4 \mathrm{v}: 6]$

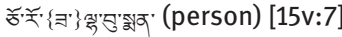

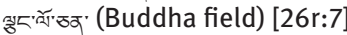

\section{ळ"}

केमत' (place) [26r:5]

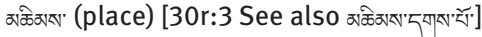

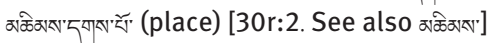

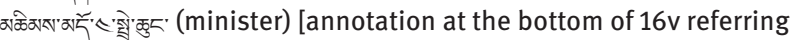
to $16 \mathrm{v}: 7]$

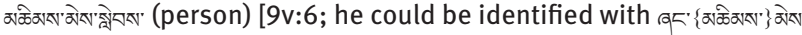
क्षेपतर, q.v.]

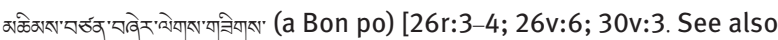

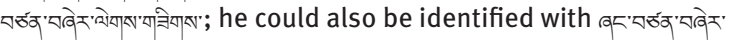
, q.v.]

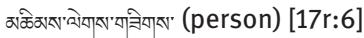

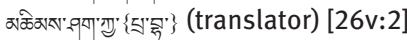

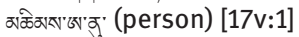

२केट:'ูु' (place) [4v:3-4] २केट'মु'वख'x' (place) [4r:3]

$E^{\prime}$

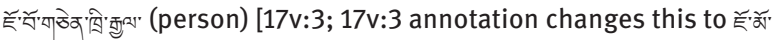

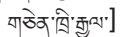

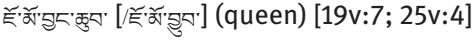

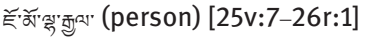

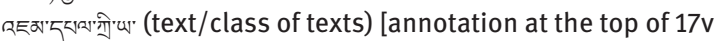
referring to $17 \mathrm{v}: 2$ ]

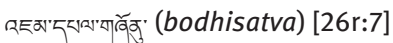

\section{ร)}

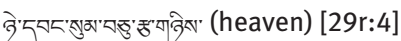

G5.' (clan) [29v:5]

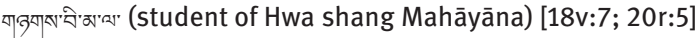

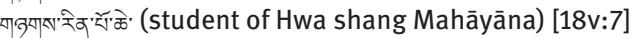

यांकर् (clan) [27r:5]

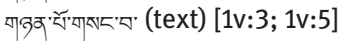

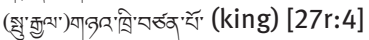

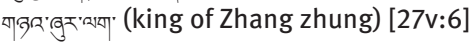

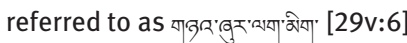

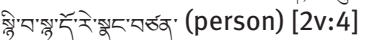

작

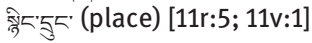

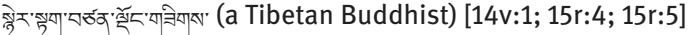

$5^{\prime}$

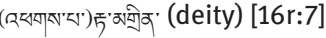

万前 5 (person) [9v:1]

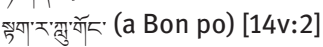

ज्ञायग' (place) [13r:3]

इ্

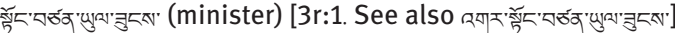

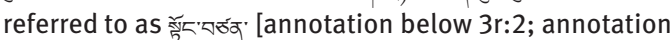
above $3 \mathrm{r}: 2$ ]

$g^{\prime}$

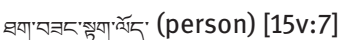

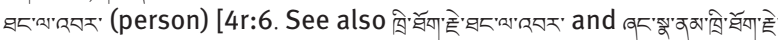
gL'arka']

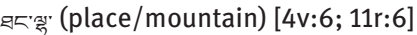

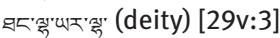

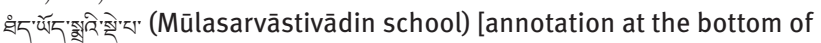
$24 \mathrm{v}$ referring to $24 \mathrm{v}: 6]$

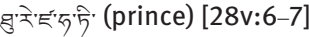

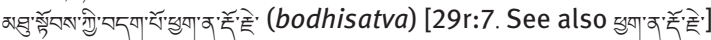

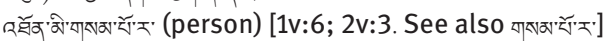

5

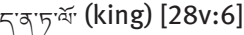

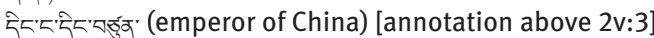

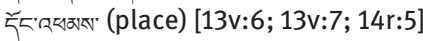

క̆av (place) [13r:3]

5 (place) [13r:3]

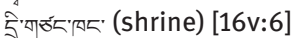

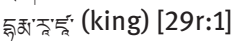

पदे'व'खव' (Buddha realm) [29r:5] 
R5व 'ख' के' (translator) [annotation at the bottom of $17 \mathrm{v}$ referring to $17 \mathrm{v}: 2$; the ara 'a added to the annotation at the top of $17 \mathrm{v}$ referring to 17v:2 may also mean this person]

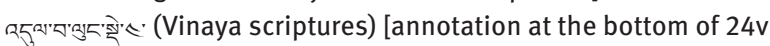
referring to $24 \mathrm{v}: 6$ ]

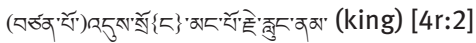

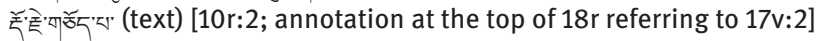

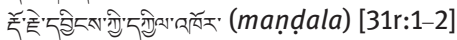

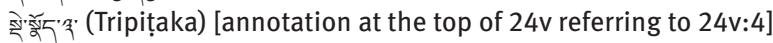

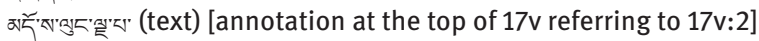

ब.

वृ'यू'सु"व (Indian Buddhist philosopher) [24v:3]

गार्वेव. (clan) [29v:5]

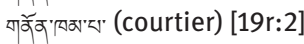

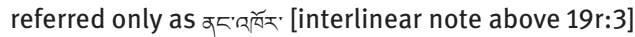

₹ख़' (translator) [25v:1]

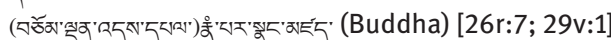

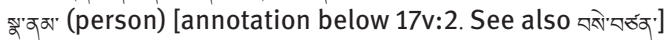

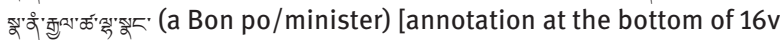
referring to $16 \mathrm{v}: 7 ; 26 \mathrm{r}: 4]$

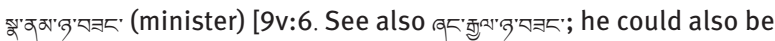

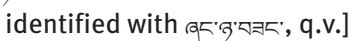

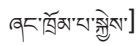

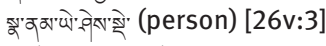

ริํำ (hot spring) [11r:3]

정저 (place) [30r:3; 30r:4]

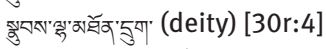

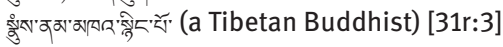

క

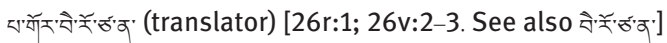

एरखब्वें (princess) [28v:7]

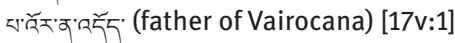

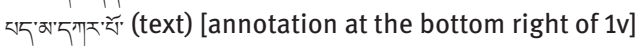

एॉ 'ख'उब्' (Buddha realm) [29r:6]

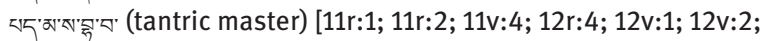

$12 \mathrm{v}: 5 ; 13 \mathrm{r}: 2 ; 14 \mathrm{v}: 5$; interlinear note below $13 \mathrm{v}: 6]$

referred to as चा अ

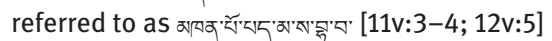

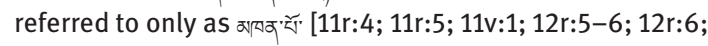

$12 \mathrm{v}: 2 ; 12 \mathrm{v}: 3 ; 12 \mathrm{v}: 4 ; 12 \mathrm{v}: 6-7 ; 13 \mathrm{r}: 4 ; 13 \mathrm{r}: 6 ; 13 \mathrm{r}: 7 ; 13 \mathrm{v}: 4$;

$13 \mathrm{v}: 5 ; 13 \mathrm{v}: 7 ; 13 \mathrm{v}: 7 ; 14 \mathrm{r}: 1 ; 14 \mathrm{r}: 2 ; 14 \mathrm{v}: 5]$

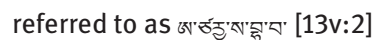

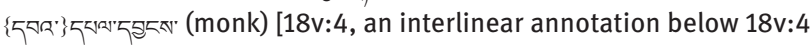
adds 5 चR:; 22v:6, an interlinear annotation below $22 \mathrm{v}: 6$ adds

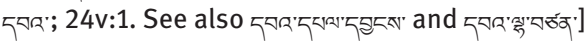

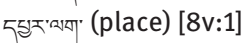

정 क्ञाल (place) [30r:1; 30r:2]

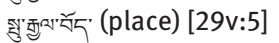

( হমব্যার' $3 \mathrm{v}: 7 ; 4 \mathrm{r}: 1]$

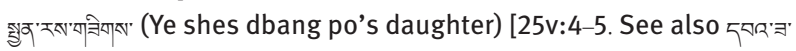

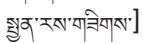

\section{ए}

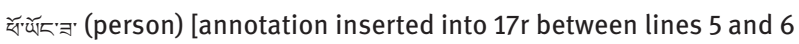
referring to $17 \mathrm{r}: 5]$

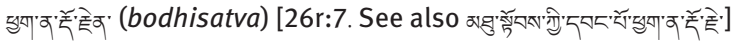

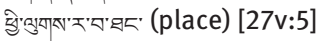

ड़्रेट'च' (fortress) [2r:1]

वस्वस:व5' (place/palace) [8r:6; 11v:4; 12r:7]

सचव ज्युत्र (place) [14v:3; 26r:5; 29v:3]

口第注' (person) [annotation inserted into $17 \mathrm{r}$ between lines 5 and 6 referring to $17 \mathrm{r}: 5$ ]

$\square^{\prime}$

पखN' (ruler of Nepal) [1v:6; 5v:5; 7r:4; 7r:5]

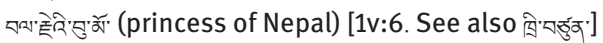

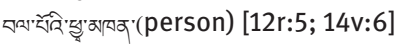

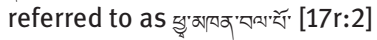

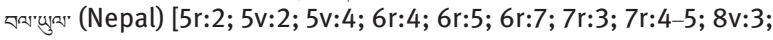
interlinear note below 8v:4; 8v:5; 10r:3; 15r:1; 19v:2; 20r:2]

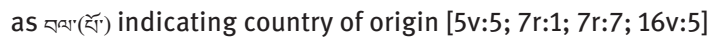

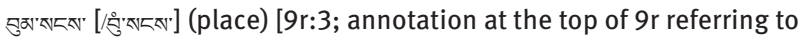
9r:2; 9r:4; 10r:7]

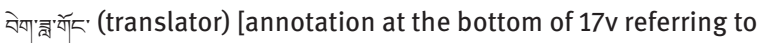
$17 \mathrm{v}: 2$; annotation at the top of $18 \mathrm{r}$ referring to $17 \mathrm{v}: 2$ ]

से:े 'छ์ ब' (translator) [17v:1; 26v:4; 26v:7; 27r:1; 28r:3; 30v:7; 31r:2;

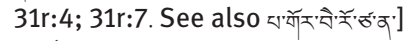

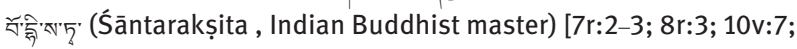
$11 \mathrm{r}: 1 ; 11 \mathrm{v}: 2 ; 12 \mathrm{v}: 1$; annotation inserted into $17 \mathrm{r}$ between lines 5 and 6 referring to $17 \mathrm{r}: 5$; interlinear note below 19v:3; 19v:5; 20r:2; 20r:4]

referred to only as ava 찬 [5v:5; 5v:7; 6r:4; 6r:6-7; 6r:7;

$7 r: 4 ; 7 r: 5 ; 7 r: 6 ; 7 r: 7 ; 7 v: 1 ; 8 r: 2 ; 8 r: 3 ; 8 r: 4 ; 8 v: 1 ; 8 v: 2 ; 8 v: 3$; $8 \mathrm{v}: 4 ; 8 \mathrm{v}: 5 ; 10 \mathrm{r}: 3 ; 10 \mathrm{r}: 4 ; 12 \mathrm{r}: 5-6 ; 14 \mathrm{r}: 7 ; 14 \mathrm{v}: 1 ; 15 \mathrm{r}: 4 ; 15 \mathrm{r}: 5$; $15 \mathrm{v}: 2 ; 15 \mathrm{v}: 3 ; 15 \mathrm{v}: 5 ; 16 \mathrm{r}$ top annotation referring to line $16 \mathrm{r}: 2 ; 16 \mathrm{r}: 4 ; 16 \mathrm{r}: 6 ; 19 \mathrm{v}: 3 ; 20 \mathrm{r}: 6]$

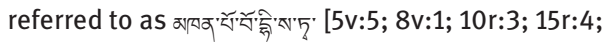
annotation at the top right of $17 \mathrm{v}$ referring to $17 \mathrm{v}: 2 ; 20 \mathrm{r}: 6]$

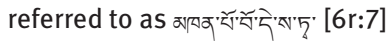

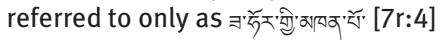

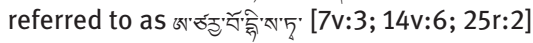

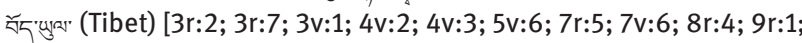
$9 \mathrm{v}: 3 ; 9 \mathrm{v}: 5 ; 10 \mathrm{v}: 6 ; 11 \mathrm{r}: 1 ; 11 \mathrm{v}: 3 ; 12 \mathrm{r}: 3 ; 13 \mathrm{r}: 3 ; 13 \mathrm{v}: 2 ; 14 \mathrm{v}: 6 ; 14 \mathrm{v}: 7$; $18 \mathrm{v}: 6 ; 19 \mathrm{v}: 1 ; 25 \mathrm{v}: 6]$

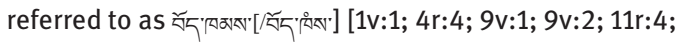
$12 r: 3 ; 12 v: 2 ; 13 r: 7 ; 13 v: 2 ; 14 r: 2 ; 14 r: 3 ; 14 r: 4 ; 25 r: 3 ; 25 v: 3$; annotation at the bottom of $25 \mathrm{v}$ referring to $25 \mathrm{v}: 6]$ referred to as خे $_{5}$. [annotation at the top of folio $2 r$ referring to $2 \mathrm{r}: 2 ; 2 \mathrm{v}: 3$; annotation at the bottom left of $4 \mathrm{r}$ referring to $4 \mathrm{r}: 6 ; 4 \mathrm{v}: 2 ; 8 \mathrm{r}: 7 ; 8 \mathrm{v}: 2 ; 9 \mathrm{r}: 2 ; 9 \mathrm{r}: 4 ; 9 \mathrm{r}: 5 ; 10 \mathrm{r}: 3 ; 10 \mathrm{r}: 3$; $12 \mathrm{r}: 3 ; 12 \mathrm{v}: 2 ; 13 \mathrm{r}: 3 ; 13 \mathrm{v}: 6 ; 14 \mathrm{v}: 7$; interlinear note below $15 \mathrm{v}: 5 ; 15 \mathrm{v}: 5 ; 15 \mathrm{v}: 6 ; 15 \mathrm{v}: 7$; annotation at the top of folio $17 \mathrm{v}$ referring to $17 \mathrm{v}: 2 ; 18 \mathrm{v}: 6 ; 19 \mathrm{r}: 7 ; 20 \mathrm{r}: 2 ; 20 \mathrm{r}: 3 ; 24 \mathrm{v}: 6$; annotation at the bottom of folio $24 \mathrm{v}$, referring to $24 \mathrm{v}: 6$; 27r:4; 29v:5; 31r:5]

इुखसरद्य (Buddha) [19r:7]

ज्ञा' द्वर: (place) [4r:3; 6r:1; 7v:1; 8r:3; 14r:6; 15r:4; 16r:2; 26r:4]

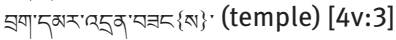

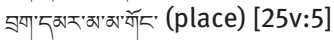

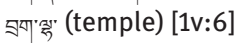

వ्रव'ग्ग'

$17 \mathrm{v}: 2$; annotation at the bottom of $17 \mathrm{v}$ referring to $17 \mathrm{v}: 2$ ]

ब्र'खि' (temple) [15r:2]

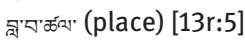

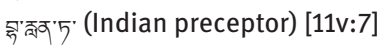

5वर्. (clan) [15r:2; 25v:3] 


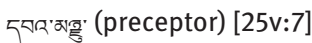

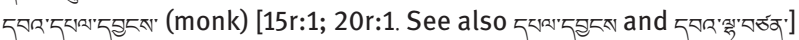

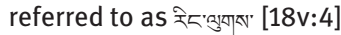

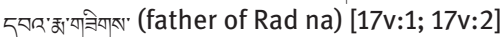

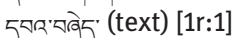

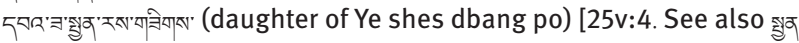
इस्ज'यद्नेगासे]

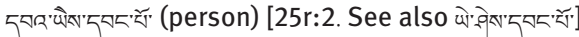

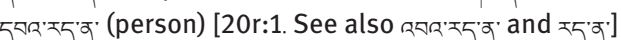

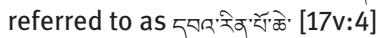

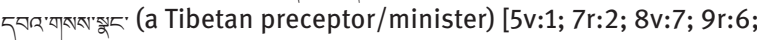

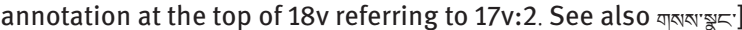

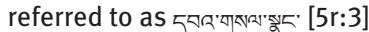

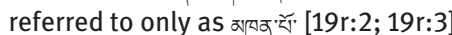

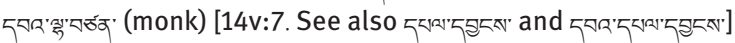

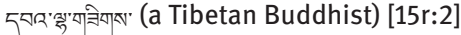

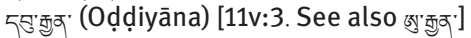

5खु'ख' (school) [22v:4-5]

5 वु के (temple/central shrine) [annotation at the top of $16 \mathrm{r}$ referring to 16r:2; 16v:1; annotation inserted into $17 \mathrm{r}$ between lines 5 and 6 referring to $17 \mathrm{r}: 5]$

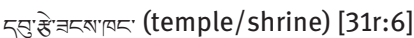

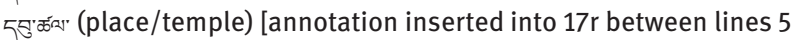
and 6 referring to $17 \mathrm{r}: 5]$

२पर:नेट (Indian preceptor) [11v:7]

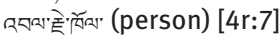

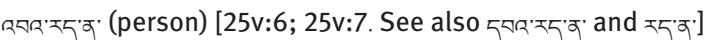

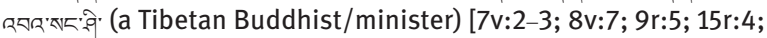
annotation at the top left of $18 \mathrm{v}$ referring to $17 \mathrm{v}: 2 ; 25 \mathrm{r}: 2 ; 26 \mathrm{r}: 1$

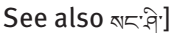

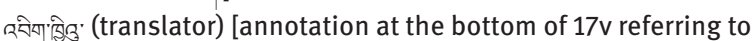
$17 \mathrm{v}: 2]$

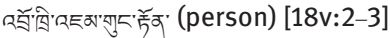

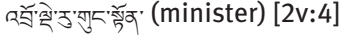

점저 (clan) [29v:5]

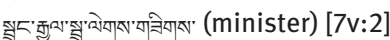

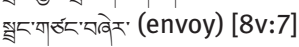

\section{$\mathbb{Q}^{\prime}$}

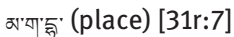

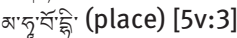

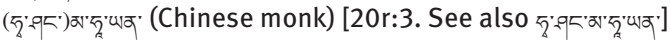

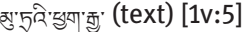

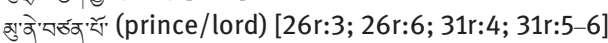

अए युㅏㅁ (place) [interlinear note below $5 \mathrm{r}: 2 ; 5 \mathrm{v}: 2 ; 5 \mathrm{v}: 4 ; 5 \mathrm{v}: 5 ; 6 \mathrm{v}: 4$; 7r:5; 7r:6; 10v:7; 14r:1]

ओसा'न'न' (spring) [12v:5]

तु बुुर' (deity) [29v:6]

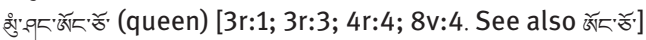

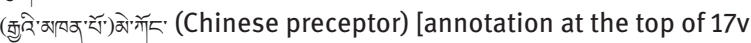
referring to $17 \mathrm{v}: 2]$

ðัवें (place) [13r:1]

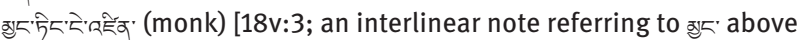
$18 \mathrm{v}: 4$ may also mean this person]

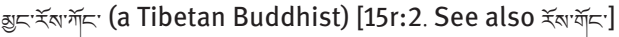

कुराइ:-'ओें' (student of Hwa shang Mahāyāna) [18v:7; 20r:4]

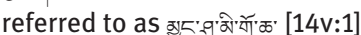

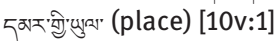

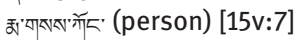

छु. (clan) [27r:5; 27r:6]

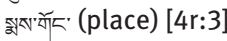

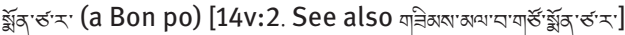

ঠ"

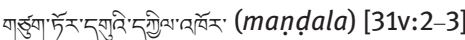

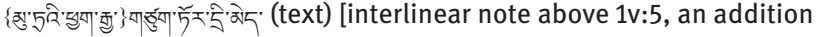

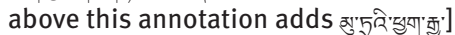

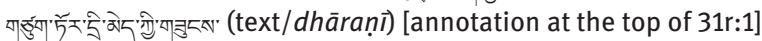

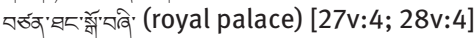

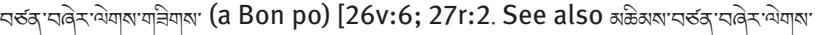

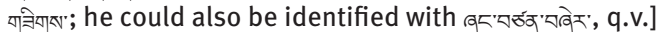

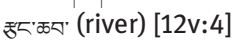

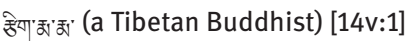

हेंख्रें (castle) [29v:7]

के

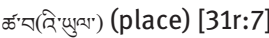

ळ"వా:

ळे (ritual) [4r:5; 4v:5; 5r:3]

के :्रे referring to $17 r: 5]$

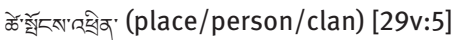

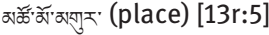

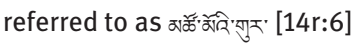

referred to as अळ" పेरिं 'रखार' [26r:4]

E.

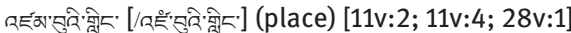

Ђ'

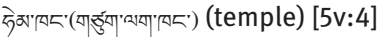

(ब)

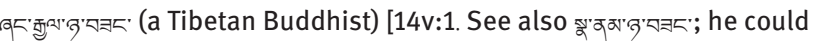

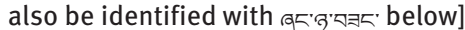

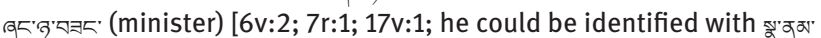

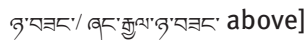

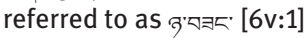

referred to as

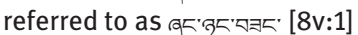

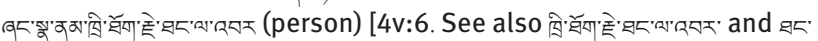
सरवस:]

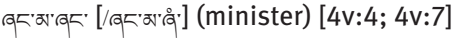

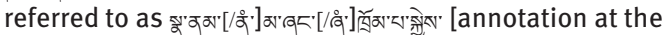
top left of $4 \mathrm{r}$ referring to $4 \mathrm{r}: 6 ; 4 \mathrm{r}: 6]$

referred to as $95^{\circ}[4 \mathrm{v}: 5]$

referred to as жrब̊: [4v:4; 4v:7]

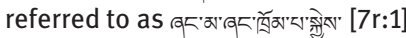

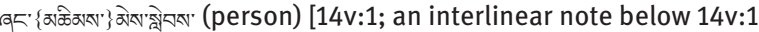

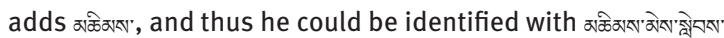
, q.v.]

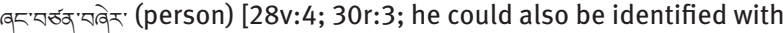

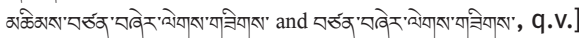

q5:gुए' (place) [27v:6; 29v:6; 29v:7]

गाबुद (place) [13r:3]

चबेस: (person) [12v:4] 


\section{ヨ’}

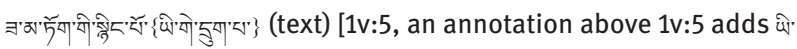

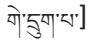

ᄏ.ॅेर' (place) [6r:6; 7r:2; 7r:4;10r:3; 20r:2]

저'या55' (ritual) [31v:5; 31v:6]

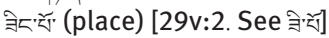

Эे:

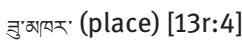

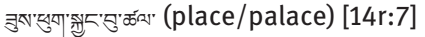

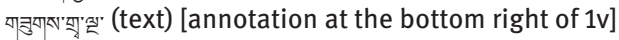

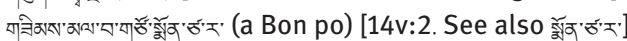

$R^{\prime}$

रंब' (place) [30r:1; 30r:2]

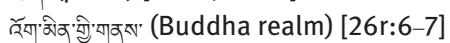

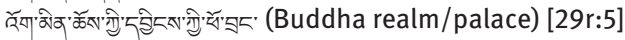

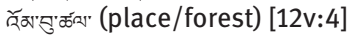

W

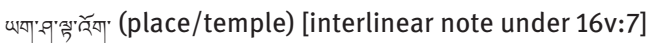

फा खे (place) [7v:4]

फर त्रुसे (river) [13v:1]

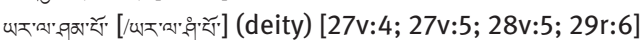

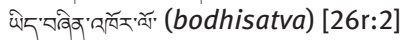

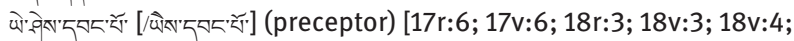

18v:5;19r:2; 19r:6; interlinear note above 19r:6; 19v:4; 24v:4;

annotation at the bottom of $24 \mathrm{v}$ referring to $24 \mathrm{v}: 6 ; 25 \mathrm{r}: 3 ; 25 \mathrm{r}: 4$;

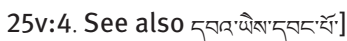

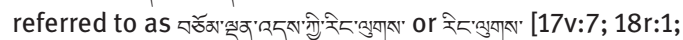

$18 \mathrm{r}: 2 ; 18 \mathrm{r}: 4]$

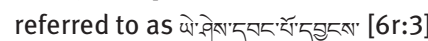

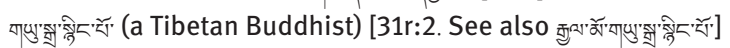

referred to as बयुु

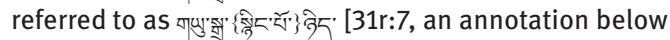

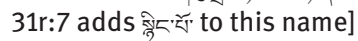

गयणुㅏ: (place) [27r:5; 27v:5]

\section{$\mathbf{X}^{\prime}$}

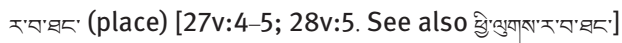

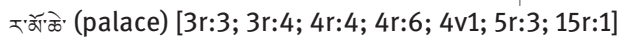

Хনत' (city) [7v:5; 12r:7]

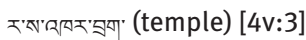

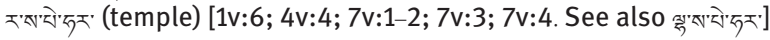

$\left\{5 \neg \Omega^{\circ}\right\} \mp 5 \cdot$ व (person) [17v:1, an annotation below $17 \mathrm{v}: 1$ adds 5 चर";

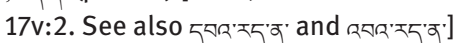

रे.х口' (Mount Meru) [12v:4; 29r:2]

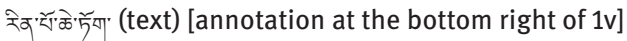

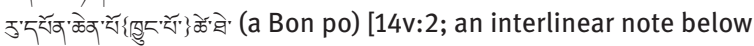

$14 \mathrm{v}: 2$ adds 迆

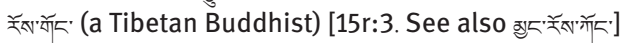

지

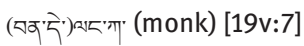

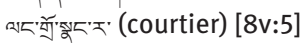

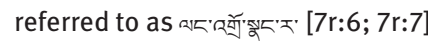

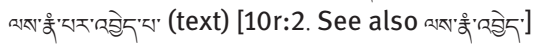

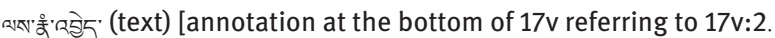

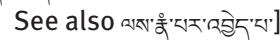

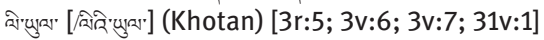

से. द्रेक (Indian scholar) [1v:7; annotation above 2r:1; 2v:3]

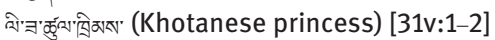

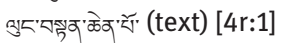

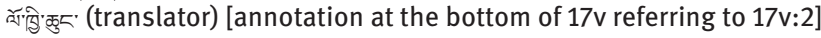

त्रदें5. (river) [6r:1]

9

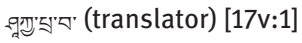

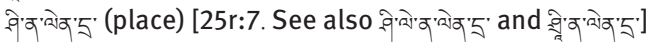

referred to as शे बेन बेन :5: [5v:3]

referred to as श्रेंब्र बेन '5: [28v:5-6]

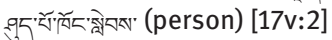

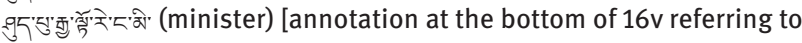
$16 \mathrm{v}: 7]$

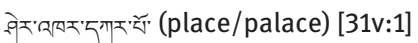

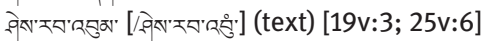

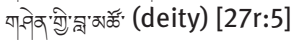

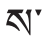

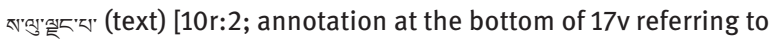
$17 \mathrm{v}: 2$; annotation at the top of $18 \mathrm{r}$ referring to $17 \mathrm{v}: 2$ ]

सा :वे' (a Tibetan Buddhist/minister) [8r:2; 9r:7; 9v:1; annotation at the top of $9 \mathrm{r}$ referring to $9 \mathrm{r}: 2 ; 9 \mathrm{v}: 2 ; 9 \mathrm{v}: 6 ; 10 \mathrm{v}: 4 ; 22 \mathrm{r}: 6$. See also

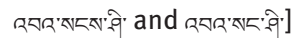

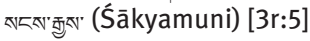

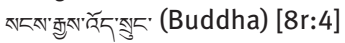

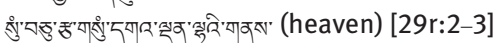

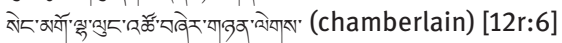

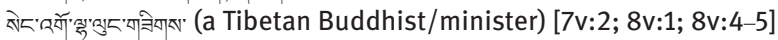

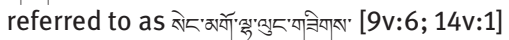

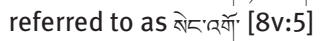

ज्ञोऽ:

त्रेपबस (ruler) [30r:3]

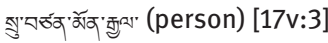

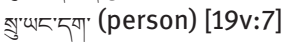

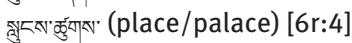

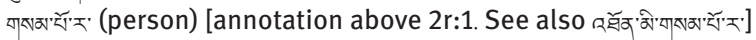

$\{5$ वर' $\}$ या।

$7 \mathrm{r}: 2 ; 7 \mathrm{r}: 4 ; 7 \mathrm{r}: 6 ; 8 \mathrm{v}: 5$, an annotation below 8v:5 adds 57q; 8v:6; annotation at the top of 9r referring to $9 \mathrm{r}: 2 ; 10 \mathrm{v}: 2 ; 10 \mathrm{v}: 4 ; 10 \mathrm{v}: 7$;

$11 \mathrm{r}: 1 ; 14 \mathrm{r}: 6 ; 15 \mathrm{r}: 1-2 ; 17 \mathrm{r}: 6$, an annotation below $17 \mathrm{r}: 6$ adds 5 चव.

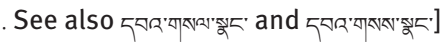

অনঅ'गा5व' ब्वेट' [19v:4]

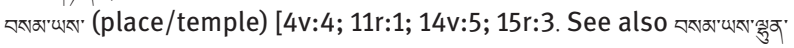

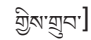

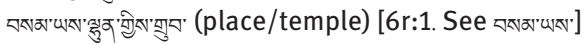

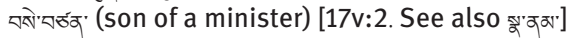

5

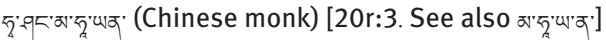
referred to only as 5:25 [19v:6; 20r:4; 20r:6; 20v:1]

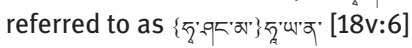

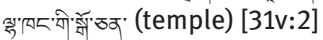

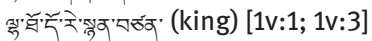

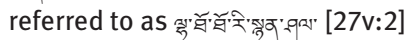




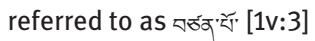

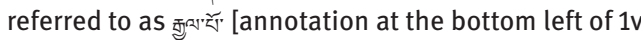
referring to $1 \mathrm{v}: 4]$

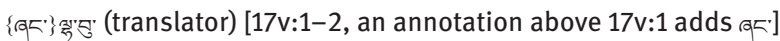

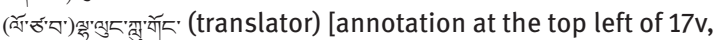
referring to $17 \mathrm{v}: 2]$

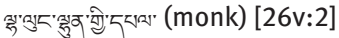

줒ㅈ' (city) [3r:3; 4r:4; 4r:6; 8r:6; 11v:4]

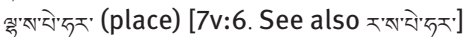

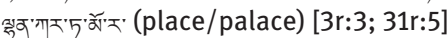

स्रेत ड्रेक्व (person) [7v:5]

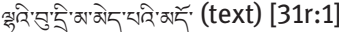

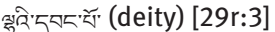

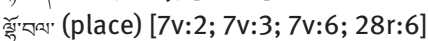

岸
$Z^{\prime}$

ऊ'वक 5. (a Kashmiri) [7v:5; 7v:6; 8r:5]

उ इे द व (deity) [16r:2; annotation at the top of 16r referring to $16 \mathrm{r}: 2]$

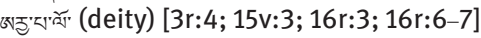

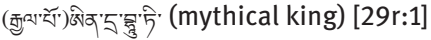

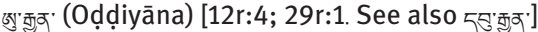

ओोग'कु' (place) [9r:1, eg chu'i 9r:2; 9r:6; 10v:4]

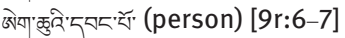

छोगा 'बेक नें' (king) [11v:7]

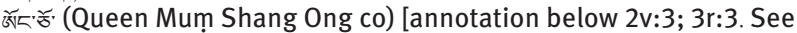

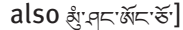

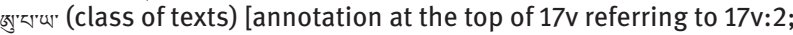
24v:6] 